\title{
Updated Outcomes of Split Course Radiotherapy in Elderly or Infirm Patients With Advanced Cancers of the Head and Neck
}

\author{
CHAIM DOMB $^{1}$, TIMOTHY D. SMILE ${ }^{1}$, CHANDANA REDDY ${ }^{1}$, NEIL M. WOODY $^{1}$, \\ SHAUNA R. CAMPBELL ${ }^{1}$, JOYCELIN F. CANAVAN ${ }^{1}$, NIKHIL P. JOSHI ${ }^{2}$, JOHN F GRESKOVICH ${ }^{3}$, \\ DEBORAH J. CHUTE ${ }^{4}$, BRIAN B. BURKEY ${ }^{5}$, LAMIE A. $\mathrm{KU}^{5}$, ERIC LAMARRE ${ }^{5}$, \\ ROBERT R. LORENZ ${ }^{5}$, BRANDON PRENDES ${ }^{5}$, JOSEPH SCHARPF ${ }^{5}$, LARISA SCHWARTZMAN ${ }^{6}$, \\ JESSICA L. GEIGER ${ }^{6}$, SHLOMO A. KOYFMAN ${ }^{1}$ and CHRISTOPHER W. FLEMING ${ }^{3}$ \\ ${ }^{1}$ Department of Radiation Oncology, Taussig Cancer Institute, Cleveland Clinic, Cleveland, OH, U.S.A.; \\ ${ }^{2}$ Department of Radiation Oncology, Rush University Cancer Center, Chicago, IL, U.S.A.; \\ ${ }^{3}$ Department of Radiation Oncology, Maroone Cancer Center, Cleveland Clinic Florida, Weston, FL, U.S.A.; \\ ${ }^{4}$ Department of Pathology, Cleveland Clinic, Cleveland, OH, U.S.A.; \\ ${ }^{5}$ Head and Neck Institute, Cleveland Clinic, Cleveland, OH, U.S.A.; \\ ${ }^{6}$ Department of Hematology and Medical Oncology, Cleveland Clinic, Cleveland, OH, U.S.A.
}

\begin{abstract}
Background/Aim: Head and neck cancers are often treated with extended courses of radiotherapy (RT), which may prove excessively toxic for frail patients. Split course RT (SCRT) delivers two courses of $R T$ separated by 4-6 weeks, personalizing treatment intensity based on response. In this study, we present our updated experience using this technique. Patients and Methods: From a single institution database, we identified patients considered for SCRT. For patients undergoing a second course of $R T$, cumulative incidence of locoregional recurrence $(L R R)$ and overall survival $(O S)$ are reported. Results: A total of 98 patients were included, of whom seventy-five percent underwent a second course of RT. The most common fractionation was $30 \mathrm{~Gy}$ in 10 fractions for each course, with a median cumulative dose of $60 \mathrm{~Gy}$. In those undergoing a second course of RT, median OS was 9.7 months and cumulative incidence of LRR at 6, 12, and 24 months was 17.0\%, 23.1\%, and 29.4\%, respectively. Conclusion: SCRT offers an attractive treatment paradigm to personalize radiation intensity based on patient tolerance, while maintaining reasonable safety and efficacy in those unfit for standard full course RT.
\end{abstract}

This article is freely accessible online.

Correspondence to: Chris Fleming, MD, Cleveland Clinic Florida, Department of Radiation Oncology, Maroone Cancer Center, 2950 Cleveland Clinic Blvd, Weston, FL 33331, U.S.A. Tel: +1 8774632010, e-mail: cfleming654@gmail.com

Key Words: Split course, radiotherapy, head and neck cancer, advanced, palliative, elderly.
Locoregional progression of head and neck cancers is a significant cause of morbidity and mortality. As such, patients with advanced disease undergo intensive locoregional therapies, generally including 6 to 7 weeks of daily RT. Treatment may be delivered either postoperatively or definitively, and often with concurrent systemic therapy $(1,2)$. Unfortunately, these prolonged courses of RT carry significant risk of toxicity even with modern treatment techniques, with $80 \%$ of patients experiencing grade 3 or higher acute adverse events (3). Toxicity is a significant challenge when treating patients with poor performance status or significant comorbidities, for whom standard treatment may be intolerable.

Split course radiation therapy (SCRT) allows for the titration of treatment intensity based on patient tolerance and disease response (4-6). In this treatment paradigm, RT is split into two courses, generally 1-2.5 weeks in length, separated by a 4-6-week treatment break. This break allows for evaluation of efficacy and tolerability before delivering the second and final course of treatment. Normal tissue recovery also occurs during this break, resulting in lower rates of acute grade $\geq 3$ toxicities of $41-53 \%(7,8)$, which may allow these patients to receive a traditional cumulative dose of 60 70 Gray (Gy) that they otherwise could not tolerate if delivered continuously. However, treatment breaks may also allow for accelerated repopulation of the malignant cells, potentially reducing efficacy of treatment and highlighting the need for appropriate patient selection (4, 5, 9). Additionally, hypofractionation of each treatment course could potentially increase late radiation effects. We have previously reported our institutional outcomes utilizing 
SCRT, finding that this paradigm was well tolerated and offered durable locoregional control (7). Herein, we present our updated institutional experience with SCRT, reporting the efficacy and safety of this approach.

\section{Patients and Methods}

From an IRB-approved single institution database of head and neck cancer patients, we identified patients treated with RT between 1999 and 2019 for primary head and neck cancers. Patients were included if they received an initial course of RT delivering 20 to 40 Gy and were documented to have been considered for a split course treatment. Patient, tumor, and treatment characteristics were recorded, as well as toxicity and efficacy endpoints. Patient characteristics included Eastern Cooperative Oncology Group performance status, sex, age, and tobacco usage. Tumor characteristics included histology, AJCC $7^{\text {th }}$ edition group stage, HPV status, primary or recurrent disease, and location. Treatment characteristics included prior surgery, RT dose, duration of treatment, laterality, treatment technique, elective nodal coverage, and use of concurrent systemic therapy. Toxicities included acute dysphagia, mucositis, dermatitis, and xerostomia. Rates of feeding tube insertion and tracheostomy were also recorded. Toxicities were determined by physician documentation and were graded according to the Common Terminology Criteria for Adverse Events v4.0.

Details of radiotherapy. As this study includes SCRT treatments over the course of two decades, treatment planning and delivery techniques were expectedly heterogeneous. During this time RT evolved from primarily forward planned 3-dimensional conformal RT (3DCRT) to inverse planned intensity modulated RT (IMRT). Currently at our institution virtually all SCRT treatments are delivered using IMRT, consisting of 10-12 daily fractions of 3 Gy for cumulative doses of 3036 Gy. For particularly symptomatic or frail patients, 20 Gy in 5 fractions may be chosen to expedite completion of treatment. Generally, all gross disease in the head and neck is treated with prescription dose in each course. Elective nodal irradiation (ENI) is often delivered during one of the two courses, typically the first, while the other course targeted gross disease only. Patients are seen in follow up 3-4 weeks after completion of the first course of RT and assessed by interval history and physical exam. Those with at least stable response to treatment and tolerable side effects are offered a second course of SCRT. Resimulation for the second course often includes imaging through the lungs to ensure the absence of distant metastatic disease. Cumulative spinal cord and brainstem maximum point dose are always kept below 2 Gy per fraction (total dose always $<50 \mathrm{~Gy}$ ).

Similarly, brachial plexus doses are kept below 2 Gy per fraction whenever possible, unless involved by gross tumor. Doses to remaining organs are as low as reasonably achievable.

Statistical analysis. For patients who underwent a second course of RT, locoregional recurrence (LRR) was estimated using competingrisks regression with death without LRR as the competing risk. Competing-risks regression was used to assess for factors associated with LRR. Overall survival (OS) and progression free survival(PFS) were estimated with Kaplan-Meier methods. PFS events included any failure as well as death. Endpoints were calculated from last date of RT. All statistical analyses were performed using SAS v9.4 (SAS Institute, Cary, NC, USA), and a $p$-value of $<0.05$ was considered statistically significant.
Table I. Patient characteristics.

\begin{tabular}{|c|c|c|}
\hline & Entire cohort & $\begin{array}{l}\text { Patients undergoing } \\
\text { both courses }\end{array}$ \\
\hline & $\mathrm{n}$ & $\mathrm{n}$ \\
\hline & 98 & 73 \\
\hline \multicolumn{3}{|l|}{ Age } \\
\hline$\leq 70$ & $44(45 \%)$ & $31(42 \%)$ \\
\hline$>70$ & $54(55 \%)$ & $42(58 \%)$ \\
\hline \multicolumn{3}{|l|}{ Smoking history } \\
\hline Current & $30(31 \%)$ & $20(27 \%)$ \\
\hline Former & $49(50 \%)$ & $38(52 \%)$ \\
\hline Never & $17(18 \%)$ & $13(18 \%)$ \\
\hline Unknown & $2(2 \%)$ & $2(3 \%)$ \\
\hline \multicolumn{3}{|l|}{ Performance status } \\
\hline 0 & $13(13 \%)$ & $9(12 \%)$ \\
\hline 1 & $55(56 \%)$ & $44(60 \%)$ \\
\hline $2+$ & $30(31 \%)$ & $20(27 \%)$ \\
\hline \multicolumn{3}{|l|}{ Disease history } \\
\hline Initial/2nd primary & $80(82 \%)$ & $58(79 \%)$ \\
\hline Recurrence & $18(18 \%)$ & $15(21 \%)$ \\
\hline \multicolumn{3}{|l|}{ Histology } \\
\hline $\mathrm{SCC}$ & $79(81 \%)$ & $61(84 \%)$ \\
\hline Other & $19(19 \%)$ & $12(16 \%)$ \\
\hline \multicolumn{3}{|l|}{ Group stage } \\
\hline I-III & $15(15 \%)$ & $10(14 \%)$ \\
\hline IV & $79(81 \%)$ & $59(81 \%)$ \\
\hline Unknown & $4(4 \%)$ & $4(5 \%)$ \\
\hline \multicolumn{3}{|l|}{ M-Stage } \\
\hline 0 & $72(73 \%)$ & $55(75 \%)$ \\
\hline 1 & $26(27 \%)$ & $18(25 \%)$ \\
\hline
\end{tabular}

SCC: Squamous cell carcinoma.

\section{Results}

Patient characteristics. Ninety-eight patients considered for SCRT were identified and included in this study. Patients were treated between 1999 and 2019. Median follow up was 5.2 months (range $=0-106$ months). Patient characteristics are summarized in Table I. Sixty-two percent of patients were male, with a median age of 72.5 (range $=36-93$ years). ECOG performance status was $\geq 2$ in $31 \%$ and $81 \%$ of patients had a history of smoking. The most common reason for SCRT was clinician-assessed inability to tolerate therapy due to poor functional status or comorbidities (96\%). The most common primary tumor sites were larynx (25\%), hypopharynx $(18 \%)$, oral cavity $(16 \%)$, and oropharynx $(15 \%)$, major salivary glands $(5 \%)$, and thyroid $(5 \%)$. The majority of patients had squamous cell carcinoma (81\%); additional histologies included adenocarcinoma (4\%), adenoid cystic (2\%), melanoma (2\%), sarcoma (2\%), undifferentiated carcinoma (2\%), and neuroendocrine carcinoma (1\%). For oropharynx tumors, 14 of 15 had 


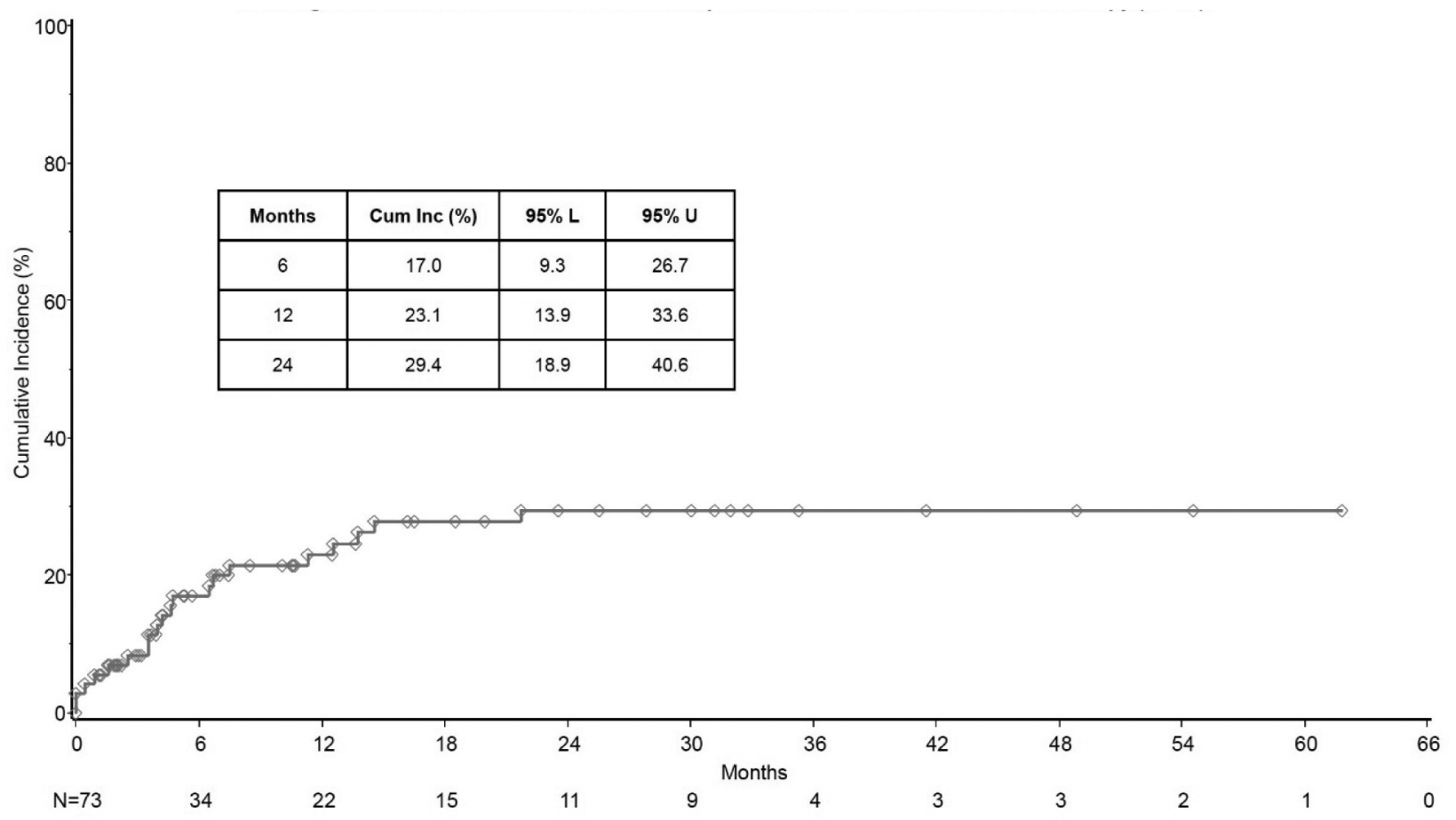

Figure 1. Locoregional recurrence for patients who completed second course of radiation therapy $(N=73)$.

known HPV status, of which $93 \%$ were HPV positive. Tumors were generally advanced, as $81 \%$ had AJCC $7^{\text {th }}$ edition stage IV disease at time of SCRT. Specifically, $18 \%$ had $\mathrm{T} 3$ disease, $45 \% \mathrm{~T} 4,43 \% \mathrm{~N} 2,10 \% \mathrm{~N} 3$, and $27 \%$ had distant metastases at time of SCRT. Eighteen percent of patients were treated for recurrent disease and $9 \%$ had received prior head and neck $\mathrm{RT}$.

Treatment. Seventy-three patients $(75 \%)$ underwent a second course of RT. Ten patients were deemed unlikely to benefit from a second course, due to either deterioration of their condition, progression of metastatic disease, or lack of tolerance to further RT. Three patients died before the second course, one did not return for follow-up, and for six the reason could not be ascertained. The most common fractionation was $30 \mathrm{~Gy}$ in 10 fractions for both first and second courses. For the first course, $14 \%$ received $<30 \mathrm{~Gy}$, $54 \%$ received $30-33$ Gy and $32 \%$ received $36-40 \mathrm{~Gy}$. For the second course, $22 \%$ received $<30$ Gy, $62 \%$ received 30 $33 \mathrm{~Gy}$, and $16 \%$ received $36-40 \mathrm{~Gy}$. The median cumulative dose was 60 Gy (range=20-72 Gy), and the median number of fractions was 20 (range $=5-44)$. A small number of patients were treated postoperatively (18\%), with concurrent chemotherapy $(6 \%)$, or with twice daily treatment of $1.5 \mathrm{~Gy}$ per fraction (6\%). Median interval between courses was 36 days (range $=21-195$ days). For first and second courses of
SCRT, IMRT was utilized in $48 \%$ and $51 \%$, respectively. Of patients who received IMRT for their initial course, $70 \%$ underwent a second course. For the 71 patients in whom laterality of SCRT was available, $87 \%$ received treatment to the bilateral neck.

Efficacy. In patients undergoing a second course of RT, cumulative incidence of LRR at 6, 12, and 24 months was $17.0 \%, 23.1 \%$, and 29.4\%, respectively (Figure 1). No factors were significantly associated with LRR (Table II). Distant progression occurred in 21.9\%. Median PFS was 5.3 months and median OS was 9.7 months, with $43.6 \%$ alive at 12 months and $24.8 \%$ at 24 months (Figure 2). In those $25 \%$ of patients who did not receive a second course of RT, median OS was 2 months. For the entire cohort, 74 patients had died at last follow up, of which $53 \%$ had documented causes of death. Of these, $72 \%$ were due to head and neck cancer and $28 \%$ to comorbidities.

Toxicity. Among the entire cohort, 23\% experienced acute grade 3-4 adverse events. No patients experienced grade 5 toxicity. Rates of acute grade 3-4 dysphagia, mucositis, dermatitis, and xerostomia were $22 \%, 6 \%, 1 \%$, and $0 \%$, respectively. Feeding tube and tracheostomy rates within 90 days of treatment were $46 \%$ and $14 \%$, respectively. Thirty-six percent had feeding tubes in place at last follow up. One patient required dilations for 


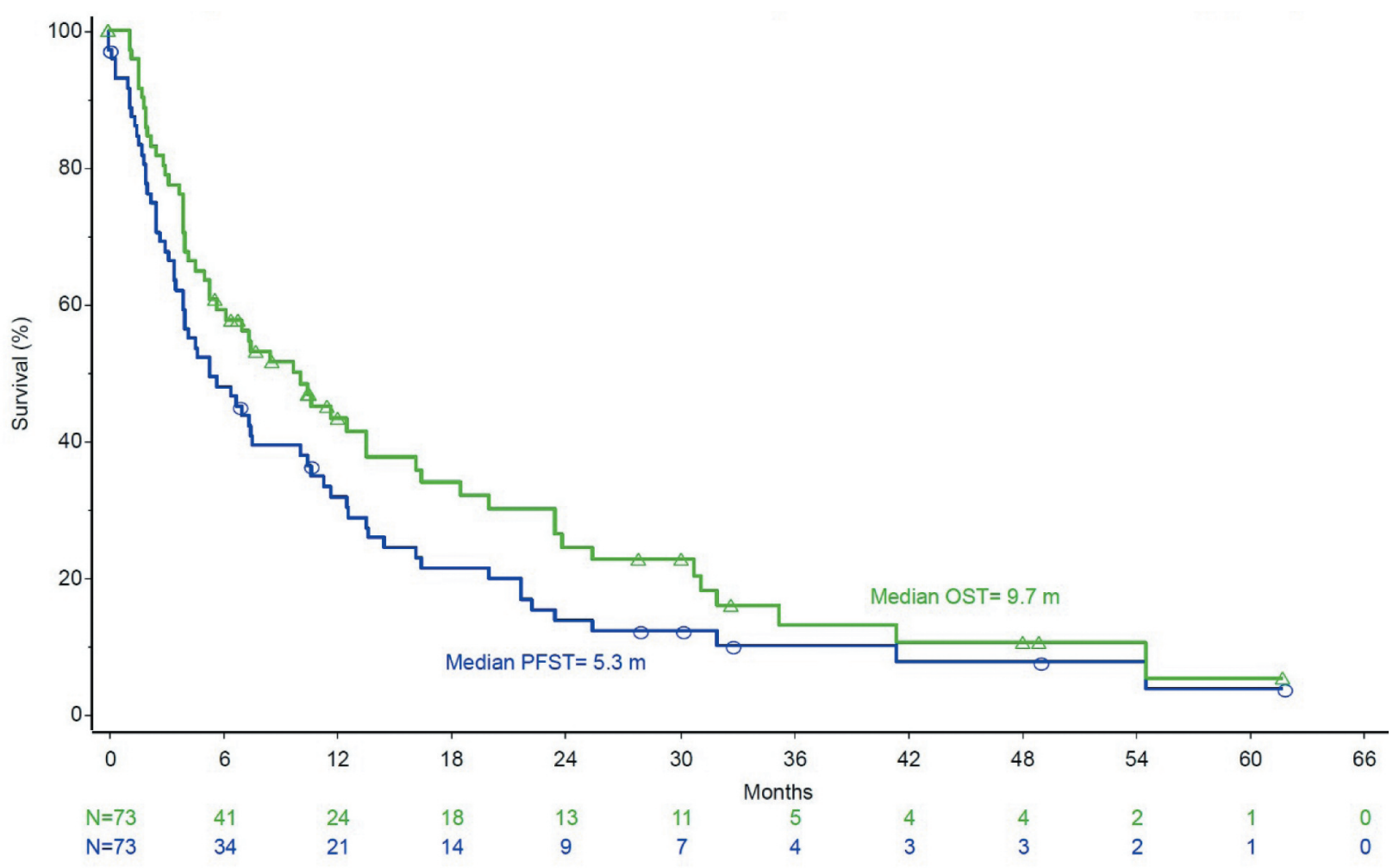

Figure 2. Progression-free and overall survival for patients who completed second course of radiation therapy $(N=73)$.

treatment-induced esophageal stricture, and there were no cases of radiation-induced brachial plexopathy.

\section{Discussion}

This study reports our updated institutional experience of patients treated with SCRT. For this population of older and poorer performance status patients with advanced head and neck cancers, one quarter did not proceed to a second course of RT, suggesting appropriate initial selection of a reduced intensity treatment approach. For patients able to complete both courses, efficacy was promising, with $23 \%$ incidence of LRR at 1 year. SCRT was tolerable, with moderate acute and late toxicity rates.

The efficacy of SCRT for head and neck cancers has been reported by several other studies. Minatel et al. reported $69 \%$ locoregional control at 7 months in patients receiving $50 \mathrm{~Gy}$ in 20 fractions with a 2-week break after the first 25 Gy (10), similar to our finding of $23 \%$ LRR at 1 year. Kancherla et al. reported outcomes after a cumulative dose of $40 \mathrm{~Gy}$ in 10 fractions with a two-week break after the first $20 \mathrm{~Gy} ; 72 \%$ of patients had at least partial response to SCRT, with 1-year PFS of $35 \%$ (9), similar to $43.6 \%$ in our study. RTOG 9003 randomized patients between 4 arms of different fractionation,
Table II. Factors associated with LRR.

\begin{tabular}{lccc}
\hline & HR & $95 \% \mathrm{CI}$ & $p$-Value \\
\hline Age ( $\leq 70$ vs. $>70)$ & 1.19 & $0.5-2.82$ & 0.70 \\
Smoking (Current $v s$. Former/Never) & 0.52 & $0.15-1.82$ & 0.30 \\
ECOG (0-1 vs. $\geq 2)$ & 1.19 & $0.44-3.18$ & 0.73 \\
Primary disease $v s$. Recurrent & 1.00 & $0.32-3.11$ & 0.99 \\
Histology (Other $v s$. SCC) & 0.55 & $0.12-2.64$ & 0.46 \\
Group stage (I-III $v s$. IV) & 0.74 & $0.19-2.91$ & 0.66 \\
M-Stage (0 vs. 1) & 3.75 & $0.95-14.78$ & 0.06 \\
Total RT dose (continuous) & 1.03 & $0.94-1.13$ & 0.54 \\
Length of treatment break (continuous) & 1.00 & $0.98-1.01$ & 0.58 \\
\hline
\end{tabular}

SCC: Squamous cell carcinoma; ECOG: Eastern Cooperative Oncology Group; RT: radiotherapy.

one of which included a split course accelerated arm, delivering 67.2 Gy over 6 weeks with a 2-week break after 38.4 Gy (11). Most arms showed roughly 30-35\% locoregional recurrence rates at one year, similar to the results of our study. However, locoregional control at 24 months was $47.8 \%$, numerically worse than $53.8 \%$ in the continuous accelerated arm of the trial, suggesting a possible detriment to treatment break with longer follow up. Indeed, many studies 
have found inferior outcomes in patients requiring treatment breaks during radiotherapy (12), highlighting the importance of not over selecting patients for SCRT. However, for patients with limited performance status and advanced disease, considerations regarding the toxicity mitigation of SCRT may override the control benefits gained with continuous treatment. Our reported median survival of only 9 months despite promising locoregional control suggests selection for SCRT was generally appropriate.

We found SCRT to be well-tolerated, with an overall rate of acute grade 3-4 adverse events of $23 \%$, with grade 3-4 dysphagia, mucositis, and dermatitis of $22 \%, 6 \%$, and $1 \%$, respectively. Similarly, Kancherla et al. reported low rates of toxicity, with $6 \%$ and $9 \%$ grade 3 mucositis and esophagitis, respectively (9). In contrast, Minetal et al. found $43 \%$ grade 3 mucositis, potentially due to concurrent use of bleomycin (10). Stadler et al. found grade 3 mucositis and dermatitis of $42 \%$ and $53 \%$, respectively (8). However, these patients received two courses of $30 \mathrm{~Gy}$ in 20 fractions, with concurrent chemotherapy, only a twoweek break between courses, and a radiotherapy boost to 70 Gy (8) - a considerably more aggressive treatment paradigm than ours. Our study has several limitations. Some patients had missing information, specifically on the cause of death, which was not unexpected given this cohort of patients had poor performance status and advanced cancers. Additionally, the suboptimal survival of many patients in this cohort limits our ability to assess long term safety and efficacy outcomes of SCRT. However, for the minority of patients who had over 1.5 years of follow up, locoregional control and toxicity outcomes are encouraging. Although the predominant cancer type was squamous cell carcinoma, a variety of other histologies were included. While this heterogeneity may potentially confound our endpoints, it also speaks to the versatility of SCRT. As the regimens used were fairly consistent, this study is also unable to answer the question of whether shorter split course regimens (e.g. $20 \mathrm{~Gy}$ in 5 fractions twice) or purely palliative regimens (e.g. quad shot) would yield similar results with less inconvenience to the patient. Finally, selection for SCRT is subjective based on each clinician's assessment of prognosis and tolerance of treatment, and therefore may be difficult to standardize. In conclusion, we found that SCRT offers an attractive treatment paradigm for advanced head and neck cancers in elderly or infirm patients, allowing clinicians to tailor intensity of RT based on response to treatment, while still maintaining safety and efficacy in those unfit for standard full course RT. Patients completing both courses of SCRT may achieve durable disease control. The inability to undergo a second course of RT portends a dismal prognosis, and those patients should be considered for referral to palliative or hospice care.

\section{Conflicts of Interest}

Jessica L. Geiger reports institutional research support from Regeneron and Genentech and an advisory board honorarium from Regeneron outside the submitted work. Shlomo A. Koyfman reports research support from Merck outside the submitted work. All other coauthors have no relevant conflicts of interest.

\section{Authors' Contributions}

Study concepts, study design, and data analysis and interpretation: Domb, Koyfman, and Fleming.

Data acquisition, quality control of data, and manuscript preparation: Domb and Fleming. Statistical analysis: Reddy. Manuscript editing and review: All Authors.

\section{References}

1 Denis F, Garaud P, Bardet E, Alfonsi M, Sire C, Germain T, Bergerot $\mathrm{P}$, Rhein B, Tortochaux $\mathrm{J}$ and Calais G: Final results of the 94-01 French Head and Neck Oncology and Radiotherapy Group randomized trial comparing radiotherapy alone with concomitant radiochemotherapy in advanced-stage oropharynx carcinoma. J Clin Oncol 22(1): 69-76, 2004. PMID: 14657228. DOI: $10.1200 / \mathrm{JCO} .2004 .08 .021$

2 Adelstein DJ, Li Y, Adams GL, Wagner H Jr, Kish JA, Ensley JF, Schuller DE and Forastiere AA: An intergroup phase III comparison of standard radiation therapy and two schedules of concurrent chemoradiotherapy in patients with unresectable squamous cell head and neck cancer. J Clin Oncol 21(1): 92-98, 2003. PMID: 12506176. DOI: 10.1200/ JCO.2003.01.008

3 Gillison ML, Trotti AM, Harris J, Eisbruch A, Harari PM, Adelstein DJ, Jordan RCK, Zhao W, Sturgis EM, Burtness B, Ridge JA, Ringash J, Galvin J, Yao M, Koyfman SA, Blakaj DM, Razaq MA, Colevas AD, Beitler JJ, Jones CU, Dunlap NE, Seaward SA, Spencer S, Galloway TJ, Phan J, Dignam JJ and Le QT: Radiotherapy plus cetuximab or cisplatin in human papillomavirus-positive oropharyngeal cancer (NRG Oncology RTOG 1016): a randomised, multicentre, non-inferiority trial. Lancet 393(10166): 40-50, 2019. PMID: 30449625. DOI: 10.1016/S0140-6736(18)32779-X

4 Agarwal JP, Nemade B, Murthy V, Ghosh-Laskar S, Budrukkar A, Gupta T, D'Cruz A, Pai P, Chaturvedi P and Dinshaw K: Hypofractionated, palliative radiotherapy for advanced head and neck cancer. Radiother Oncol 89(1): 51-56, 2008. PMID: 18649963. DOI: 10.1016/j.radonc.2008.06.007

5 Chen AM, Vaughan A, Narayan S and Vijayakumar S: Palliative radiation therapy for head and neck cancer: toward an optimal fractionation scheme. Head Neck 30(12): 1586-1591, 2008. PMID: 18798313. DOI: 10.1002/hed.20894

6 Grewal AS, Jones J and Lin A: Palliative radiation therapy for head and neck cancers. Int J Radiat Oncol Biol Phys 105(2): 254-266, 2019. PMID: 31128145. DOI: 10.1016/j.jirobp.2019.05.024

7 Bledsoe TJ, Noble AR, Reddy CA, Burkey BB, Greskovich JF, Nwizu T, Adelstein DJ, Saxton JP and Koyfman SA: Split-course accelerated hypofractionated radiotherapy (SCAHRT): A safe and effective option for head and neck cancer in the elderly or infirm. Anticancer Res 36(3): 933-939, 2016. PMID: 26976981. 
8 Stadler P, Putnik K, Kreimeyer T, Sprague LD, Koelbl O and Schäfer C: Split course hyperfractionated accelerated radiochemotherapy (SCHARC) for patients with advanced head and neck cancer: influence of protocol deviations and hemoglobin on overall survival, a retrospective analysis. BMC Cancer 6: 279, 2006. PMID: 17150114. DOI: 10.1186/1471-2407-6-279

9 Kancherla KN, Oksuz DC, Prestwich RJ, Fosker C, Dyker KE, Coyle CC and Sen M: The role of split-course hypofractionated palliative radiotherapy in head and neck cancer. Clin Oncol ( R Coll Radiol) 23(2): 141-148, 2011. PMID: 20934860. DOI: 10.1016/j.clon.2010.09.006

10 Minatel E, Gigante M, Franchin G, Gobitti C, Mascarin M, Bujor L, Barzan L and Trovò MG: Combined radiotherapy and bleomycin in patients with inoperable head and neck cancer with unfavourable prognostic factors and severe symptoms. Oral Oncol 34(2): 119-122, 1998. PMID: 9682774. DOI: 10.1016/ s1368-8375(97)00073-0
11 Beitler JJ, Zhang Q, Fu KK, Trotti A, Spencer SA, Jones CU, Garden AS, Shenouda G, Harris J and Ang KK: Final results of local-regional control and late toxicity of RTOG 9003: a randomized trial of altered fractionation radiation for locally advanced head and neck cancer. Int J Radiat Oncol Biol Phys 89(1): 13-20, 2014. PMID: 24613816. DOI: 10.1016/j.jijobp.2013.12.027

12 Xiang M, Gensheimer MF, Pollom EL, Holsinger FC, Colevas AD, Le QT and Beadle BM: Prolongation of definitive head and neck cancer radiotherapy: Survival impact and predisposing factors. Radiother Oncol 156: 201-208, 2021. PMID: 33383061. DOI: $10.1016 /$ j.radonc.2020.12.025

Received August 16, 2021

Revised September 1, 2021

Accepted September 2, 2021 